Pacific Journal of Mathematics

NEARLY BOREL SETS AND PRODUCT MEASURES 


\title{
NEARLY BOREL SETS AND PRODUCT MEASURES
}

\author{
ROY A. JOHNSON
}

\begin{abstract}
We examine those Borel measures which are inner regular with respect to the class of bounded sets containing the closure of each of their countable subsets. It is shown that such a Borel measure can be extended to a measure on the sigma-ring generated by that class of sets. Applications are given to the product of two Borel measures.
\end{abstract}

1. Introduction. Throughout this paper $X$ and $Y$ will be locally compact spaces (all topological spaces will be assumed to be Hausdorff spaces), and $\mu$ and $\nu$ will be Borel measures on $X$ and $Y$, respectively. In other words, $\mu$ is a countably additive, nonnegative measure on the sigma-ring generated by the class of compact sets in $X$, and each compact set has finite $\mu$-measure. For undefined measure theoretic terminology, the reader is referred to [4]. A subset of $X$ will be called nearly closed or $n$-closed if it contains the closure (in $X$ ) of each of its countable subsets; the complement of an $n$-closed set will be called nearly open or n-open. In view of the Axiom of Choice, the $n$-closed sets of a first countable space are in fact closed sets. Hence, the focus of this paper is on spaces which are not first countable; in particular, the topological groups of interest will be the nonmetrizable groups.

Recall that a set is bounded or relatively compact if its closure is compact. Every bounded, $n$-closed set is $\omega$-bounded in the sense of $[3$, p. 201], although not every $\omega$-bounded set is bounded or even sigma-bounded. In keeping with [8, §2], a class of sets is called a compact class if for each sequence in the class, the nonempty intersection for each $n$ of the first $n$ terms of the sequence implies that the intersection of the sequence is nonempty. The class of bounded $n$-closed sets is particularly important for this paper because of the following theorem which shows that this class is a compact class.

THEOREM 1.1. If $\left\{C_{i}\right\}$ is a sequence of bounded n-closed sets having the finite intersection property, then $\cap C_{i}$ is nonempty.

Proof. Since the intersection of a family of $n$-closed sets is $n$-closed, we may assume without loss of generality that $\left\{C_{i}\right\}$ is a decreasing sequence of nonempty, bounded $n$-closed sets. For each $i$, choose $x_{i} \in C_{i}$. Notice that $\left\{x_{n}: n \geqq i\right\} \subset C_{i}$ for each $i$. Let $B_{i}=$ closure $\left(\left\{x_{n}: n \geqq i\right\}\right)$. Since $C_{i}$ is $n$-closed, we see that $B_{i} \subset C_{i}$ for each 
$i$. Since each $C_{i}$ is bounded, each $B_{i}$ is thus compact. The sets $B_{i}$ form a decreasing sequence of nonempty compact sets, so that $\cap B_{i}$ and hence $\cap C_{i}$ is nonempty.

The Borel measure $\mu$ will be called regular if for each Borel set $E$,

$$
\mu(E)=\sup \{\mu(K): K \text { is a compact subset of } E\} \text {. }
$$

We shall say that $\mu$ is nearly regular or $n$-regular if for each Borel set $E$,

$$
\mu(E)=\sup \{\mu(C): C \subset E \text { and } C \text { is a bounded, } n \text {-closed Borel set }\} .
$$

Every regular Borel measure is clearly $n$-regular, but $n$-regular Borel measures can fail to be regular as we shall see later.

Just as the class of Borel sets of $X$ is the sigma-ring generated by the compact sets in $X$, so it will be convenient to define the class of nearly Borel sets or $n$-Borel sets of $X$ to be the smallest sigmaring containing the bounded $n$-closed sets in $X$. Clearly, every Borel set is an $n$-Borel set. A nearly Borel measure or $n$-Borel measure on $X$ is a countably additive, nonnegative measure on the $n$-Borel sets such that each compact set has finite measure. An $n$-Borel measure will be called nearly regular or n-regular if it is inner regular with respect to the class of bounded $n$-closed sets. That is, an $n$-Borel measure $\mu^{\prime}$ is $n$-regular if for each $n$-Borel set $E$,

$$
\mu^{\prime}(E)=\sup \left\{\mu^{\prime}(C): C \text { is a bounded } n \text {-closed subset of } E\right\} \text {. }
$$

In order to illustrate these definitions and ideas in the context of a nonmetrizable compact group, we have the following:

EXAmple 1.2. Let $\left\{X_{i}: i \in I\right\}$ be an uncountable collection of nontrivial compact groups, and let $X$ be the product of the $X_{i}$ 's. Let us say that two members of $X$ are equivalent if they agree for all but countably many coordinates. That is, $x \sim y$ if and only if $\left\{i \in I: x_{i} \neq y_{i}\right\}$ is countable. If $[x]$ denotes the equivalence class containing $x$, then it is easy to see that $[x]$ is a bounded $n$-closed set and that $[x]$ is not compact. Now fix $w \in X$. If $y \in X$, let

$$
W(y)=\left\{x \in X: \text { for each } i \in I, x_{i}=y_{i} \text { or } x_{i}=w_{i}\right\} \text {. }
$$

Define a partial order $\leqq_{w}$ on $X$ by writing $x \leqq_{w} y$ if and only if $x \in W(y)$. Fix $z \in X-[w]$, and let $\nu^{\prime}$ be the $n$-Borel measure defined on $X$ as follows: $\nu^{\prime}(E)=1$ if there exists an $n$-closed set $F$ such that $E$ contains $F \cap[w]$ and such that $z$ is a limit point of $F \cap$ $W(z) \cap[w]$; otherwise, $\nu^{\prime}(E)=0$. (Cf. [5, Example 3] or [4, Exercise 52.10].) Incidentally, for an $n$-closed set $F$, the condition that $z$ be 
a limit point of $F \cap W(z) \cap[w]$ is equivalent to the condition that for each $x \in W(z) \cap[w]$, there exists $y \in F \cap W(z) \cap[w]$ such that $x \leqq_{w} y$. The $n$-Borel measure $\nu^{\prime}$ is evidently $n$-regular. I do not know if the Borel measure $\nu$ obtained by restricting $\nu^{\prime}$ to the Borel sets of $X$ is an $n$-regular Borel measure, but that possibility seems doubtful.

Now even if we wanted to restrict our attention to Borel sets rather than $n$-Borel sets, we would find that the Borel measure $\nu$ in Example 1.2 can lead to $n$-closed sets which are not Borel sets. To see this, let $K$ be the (necessarily compact) subset of $X \times X$ given by $K=\left\{(x, y): x \leqq_{w} y\right\}$. Since $K_{x}=\left\{y: x \leqq_{w} y\right\}$, we see that $\nu\left(K_{x}\right)=1$ if $x \in W(z) \cap[w]$ and $\nu\left(K_{x}\right)=0$ otherwise. Hence, $\left\{x \in X: \nu\left(K_{x}\right)=1\right\}$ is precisely the set $W(z) \cap[w]$, and this set is not a Borel set even though it is bounded and $n$-closed. Indeed, since $W(z)$ is obviously topologically equivalent to a compact group, we may let $\lambda$ be the Borel measure on $W(z)$ corresponding to Haar measure on that compact group. If $\mu(E)=\lambda(E \cap W(z))$ for each Borel set $E$ in $X$, then the set $\left\{x \in X: \nu\left(K_{x}\right)=1\right\}$ is not even measurable with respect to the completion of the Borel measure $\mu$. (Cf. [5, Example 3].) On the other hand, Theorem 2.6 will show that this measure $\mu$ can be extended to an $n$ regular $n$-Borel measure $\mu^{\prime}$ on $X$. In that case, a product of $\mu$ and $\nu$ can be defined on the Borel sets of $X \times X$ by the formula $\rho(M)=$ $\int \nu\left(M_{x}\right) d \mu^{\prime}$ for all Borel sets $M$ in $X \times X$ [6, Theorem 4.4].

Finally, I wish to express my thanks to the referee for valuable comments concerning this paper.

2. Nearly regular nearly Borel measures. The main result of this section is the extension of an $n$-regular Borel measure to an $n$-regular $n$-Borel measure in Theorem 2.6. Because of the similarities between $n$-regular Borel measures and regular Borel measures, it will be convenient to look at these ideas in the context of the abstract system given in [1]. Throughout this section we shall therefore consider an abstract system $\{\mathscr{K}, \mu, \mathscr{C}, \mathscr{U}\}$ on a set $X$ satisfying the following axioms:

I. $\mathscr{R}$ is a ring of subsets of $X$, and $\mu$ is a finitely additive measure on $\mathscr{R}$.

II. $\mathscr{C}$ is a family of subsets of $X$, and $\mathscr{C}$ is closed under countable intersections and finite unions. We assume that the empty set is in $\mathscr{C}$ and that each member of $\mathscr{C}$ is contained in some member of $\mathscr{R}$ having finite $\mu$-measure.

III. $\mathscr{C}$ is a family of subsets of $X$, and $\mathscr{U}$ is closed under countable unions and finite intersections. We assume that if $E \in \mathscr{R}$, then there exists $U$ in $\mathscr{U}$ such that $E \subset U$, and we assume that if $U \in \mathscr{U}$, 
then there exists $F \in \mathscr{R}$ such that $U \subset F$.

IV. If $U \in \mathscr{Q}$ and $C \in \mathscr{C}$, then $U-C \in \mathscr{Q}$.

V. If $C \in \mathscr{C}$ then there exist $U \in \mathscr{C}$ and $D \in \mathscr{C}$ such that $C \subset$ $U \subset D$.

VI. If $C \in \mathscr{C}$ and $U \in \mathbb{Z}$, then $C-U \in \mathscr{C}$.

VII. The ring $\mathscr{R}$ is contained in $\mathscr{P}(\mathscr{C})$, the sigma-ring generated by $\mathscr{C}$.

These are the axioms of $[1, \S \S 59$ and 61$]$ except that we let $\mu$ be a finitely additive measure on a ring contained in $\mathscr{S}(\mathscr{C})$ rather than a countably additive measure on $\mathscr{S}(\mathscr{C})$, and the families $\mathscr{C}$ and $\mathscr{U}$ are not required to be contained in $\mathscr{R}$.

For this paper, an important example of a system satisfying Axioms I-VII is the following: Let $X$ be a locally compact space, let $\mathscr{R}$ be a ring containing the Borel sets of $X$ and contained in the $n$ Borel sets of $X$, let $\mu$ be a measure on $\mathscr{R}$ such that each compact set has finite measure, let $\mathscr{C}$ be the class of bounded $n$-closed sets in $X$, and let $\mathscr{C}$ be the class of sigma-bounded $n$-open sets. It is easy to see that Axioms I to VII are satisfied for this system $\{\mathscr{R}, \mu, \mathscr{C}, \mathscr{C}\}$.

Suppose now that $\{\mathscr{R}, \mu, \mathscr{C}, \mathscr{C}\}$ is a system satisfying Axioms I to VII. If $A$ is a subset of a member of $\mathscr{R}$, the inner measure of $A$ is given by $\mu_{i}(A)=\sup \{\mu(E): E \subset A$ and $E \in \mathscr{R}\}$, and the outer measure (exterior measure) of $A$ is given by $\mu_{e}(A)=\inf \{\mu(F): A \subset F$ and $F \in \mathscr{R}$ \}. We say that a set $E$ in $\mathscr{R}$ is inner regular if

$$
\mu(E)=\sup \left\{\mu_{i}(C): C \subset E \text { and } C \in \mathscr{C}\right\} .
$$

Equivalently, the set $E$ is inner regular if for each $k<\mu(E)$, there exists a set $F$ in $\mathscr{R}$ and a set $C$ in $\mathscr{C}$ such that $F \subset C \subset E$ and such that $k<\mu(F)$. We say that a set $E$ in $\mathscr{R}$ is outer regular if

$$
\mu(E)=\inf \left\{\mu_{e}(U): E \subset U \text { and } U \in \mathscr{C}\right\} .
$$

Notice that inner [outer] regularity of a set depends not only on the measure $\mu$ and the class $\mathscr{C}[\mathscr{U}]$ but also on the ring $\mathscr{R}$. In other words, suppose that $\mathscr{R}_{1}$ is a subring of $\mathscr{R}_{2}$ and that the measure $\mu_{1}$ on $\mathscr{R}_{1}$ is the restriction of $\mu_{2}$ to $\mathscr{R}_{1}$. If a set $E$ in $\mathscr{R}_{1}$ is inner regular for the system $\left\{\mathscr{R}_{2}, \mu_{2}, \mathscr{C}, \mathscr{C}\right\}$, it does not follow that $E$ is inner regular for the system $\left\{\mathscr{R}_{1}, \mu_{1}, \mathscr{C}, \mathscr{U}\right\}$. However, if $E$ is inner regular with respect to $\mu_{1}$, then it is also inner regular with respect to the extension $\mu_{2}$.

Since the families $\mathscr{C}$ and $\mathscr{C}$ are not assumed to be contained in $\mathscr{R}$, it is usually necessary to talk about $\mu_{i}(C)$ and $\mu_{e}(U)$ when considering inner regularity or outer regularity. However, if $\mu$ is a countably additive measure on a sigma-ring $\mathscr{R}$ and if each set in $\mathscr{R}$ is inner regular, then for each $E \in \mathscr{R}$, 


$$
\mu(E)=\sup \{\mu(C): C \subset E, C \in \mathscr{C}, \text { and } C \in \mathscr{R}\} .
$$

This assertion is proved in [8, Theorem 4(iv)]. Similarly, if $\mu$ is a countably additive measure on a sigma-ring $\mathscr{R}$ and if each set in $\mathscr{R}$ is outer regular, then for each $E \in \mathscr{R}$,

$$
\mu(E)=\inf \{\mu(U): E \subset U, U \in \dddot{\ell}, \text { and } U \in \mathscr{R}\} .
$$

THEOREM 2.1. Suppose $\{\%, \mu, \lessdot, \not 6\}$ is a system satisfying Axioms I-VII.

Then: (1) If $E$ and $F$ are disjoint inner regular [outer regular] sets in $\mathscr{R}$, then $E \cup F$ is inner regular [outer regular] also. (Cf. [1, p. 189].)

(2) If $E$ and $F$ are inner regular [outer regular] sets of finite measure in $\mathscr{R}$, then $E \cap F$ is inner regular [outer regular] also. (Cf. [1, Theorem 59.6 and p. 191].)

(3) If $\mu$ is countably additive and $\left\{E_{i}\right\}$ is an increasing sequence of inner regular [outer regular] sets in $\mathscr{R}$ and $\cup E_{i} \in \mathscr{R}$, then $\cup E_{i}$ is inner regular [outer regular] also. (Cf. [1, Theorems 59.5 and 59.4].)

(4) If $\mu$ is countably additive and $\left\{E_{i}\right\}$ is a decreasing sequence of inner regular [outer regular] sets of finite measure in $\mathscr{R}$ and $\cap E_{i} \in \mathscr{R}$, then $\cap E_{i}$ is inner regular [outer regular] also. (Cf. [1, Theorems 59.6 and 59.7].)

A set will be called "bounded" if it is a subset of some member of $\mathscr{C}$ [1, p. 195]. In view of Axiom V, every "bounded" set is contained in some "bounded" set of $\mathbb{Z}$. In view of Axiom VII, each set in $\mathscr{R}$ can be expressed as the union of a sequence of "bounded" sets in $\mathscr{R}$.

TheOREM 2.2. Suppose $\{\not{C}, \mu, \mathscr{C}, \mathscr{C}\}$ is a system satisfying Axioms I-VII and that $\mu$ is countably additive. Each set in $\mathscr{R}$ is inner regular if and only if each set in $\mathscr{R}$ is outer regular.

Proof. Suppose each set in $\mathscr{R}$ is inner regular, and suppose $E$ is a "bounded" set in $\mathscr{R}$. Choose a "bounded" set $U$ in 26 and a set $F$ in $\mathscr{R}$ such that $E \subset U \subset F$, where $\mu(F)$ is finite. Given $\varepsilon>0$, choose $G \in \mathscr{C}$ such that $G \subset F-E$ and such that $\mu_{i}(G)>\mu(F-E)-\varepsilon$. Then $E \subset U-G$, the set $U-G$ is in $\mathscr{L}$, and $\mu(E)>\mu_{e}(U-G)-\varepsilon$. Hence, each "bounded" set in $\mathscr{R}$ is outer regular, so that each set in $\mathscr{R}$ is outer regular by Theorem 2.1.

Now suppose each set in $\mathscr{R}$ is outer regular, and suppose $E$ is a "bounded" set in $\mathscr{R}$. Then there exists a set $C \in \mathscr{C}$ and a set $F$ 
in $\mathscr{R}$ such that $E \subset C \subset F$, where $\mu(F)$ is finite. Given $\varepsilon>0$, choose $U \in \mathscr{U}$ such that $F-E \subset U$ and such that $\mu(F-E)>\mu_{e}(U)-\varepsilon$. Then $C-U \subset E$, the set $C-U \in \mathscr{C}$, and $\mu(E)<\mu_{i}(C-U)+\varepsilon$. By Theorem 2.1 each set in $\mathscr{R}$ is therefore inner regular.

In the context of $n$-regular Borel measures, Theorem 2.2 tells us that a Borel measure $\mu$ is $n$-regular if and only if

$$
\mu(E)=\inf \{\mu(U): E \subset U \text { and } U \text { is an } n \text {-open Borel set }\}
$$

for each Borel set $E$.

THEOREM 2.3. Suppose $\{\mathscr{R}, \mu, \mathscr{C}, \mathscr{C}\}$ is a system satisfying Axioms I-VII and suppose, moreover, that $\mathscr{C}$ is a compact class. If each set in $\mathscr{R}$ is inner regular, then $\mu$ is countably additive. If $\mu^{\prime}$ is the unique extension of $\mu$ to a countably additive measure on the smallest sigma-ring $\mathscr{S}(\mathscr{R})$ containing $\mathscr{R}$, then each set in $\mathscr{P}(\mathscr{R})$ is inner regular (with respect to the system $\left\{\mathscr{S}(\mathscr{R}), \mu^{\prime}\right.$, $\mathscr{C}, \mathscr{C}\})$.

Proof. Use [8, Theorems 4(i) and 4(ii)].

Lemma 2.4. Let $\{\mathscr{R}, \mu, \mathscr{C}, \mathscr{C}\}$ be a system satisfying Axioms I-VII. Suppose each member of $\mathscr{R}$ is inner regular and that $C$ is a member of $\mathscr{C}$ which is not in the ring $\mathscr{R}$. If $\mathscr{R}^{\prime}$ is the smallest ring containing the set $C$ and the ring $\mathscr{R}$, then $\mu$ can be extended to a measure $\mu^{\prime}$ on $\mathscr{R}^{\prime}$ such that each member of $\mathscr{R}^{\prime}$ is inner regular (with respect to the system $\left\{\mathscr{R}^{\prime}, \mu^{\prime}, \mathscr{C}, \mathscr{C}\right\}$ ).

Proof. If $E \in \mathscr{R}^{\prime}$, let $\mu^{\prime}(E)=\mu_{e}(E \cap C)+\mu_{i}(E-C)$, where $\mu_{e}$ and $\mu_{i}$ are outer measure and inner measure, resp. Then $\mu^{\prime}$ is a measure extending $\mu$ [7, Theorem 1]. In order to show that each member of $\mathscr{R}^{\prime}$ is inner regular, it suffices to show that $(E \cap C) \Delta(F-C)$ is inner regular whenever $E, F \in \mathscr{R}$ and $\mu(E)<\infty[7$, p. 268]. If $F \in \mathscr{R}$, let us check that $F-C$ is inner regular. We wish to show that

$$
\mu^{\prime}(F-C)=\sup \left\{\left(\mu^{\prime}\right)_{i}(D): D \subset F-C \text { and } D \in \mathscr{C}\right\} .
$$

To that end, suppose $k<\mu^{\prime}(F-C)$. Since $\mu^{\prime}(F-C)=\mu_{i}(F-C)$, we may choose $G \in \mathscr{R}$ such that $G \subset F-C$ and such that $k<\mu(G)$. Since each set in $\mathscr{R}$ is inner regular, there exists a set $D$ in $\mathscr{C}$ such that $D \subset G$ and such that $k<\mu_{i}(D) \leqq\left(\mu^{\prime}\right)_{i}(D)$. It follows that

$$
\mu^{\prime}(F-C)=\sup \left\{\left(\mu^{\prime}\right)_{i}(D): D \subset F-C \text { and } D \in \mathscr{C}\right\},
$$

so that $F-C$ is inner regular. Of course, $E \cap C$ is inner regular 
by part (2) of Theorem 2.1, so that $(E \cap C) \Delta(F-C)$ is inner regular by part (1) of Theorem 2.1.

THEOREM 2.5. Let $\{\mathscr{R}, \mu, \mathscr{C}, \mathscr{U}\}$ be a system satisfying Axioms I-VII, and suppose $\mathscr{C}$ is a compact class. Let $\mathscr{S}(\mathscr{C})$ be the smallest sigma-ring containing $\mathscr{C}$. If each member of $\mathscr{R}$ is inner regular, then $\mu$ can be extended to a countably additive measure $\mu^{\prime}$ on $\mathscr{S}(\mathscr{C})$ such that each member of $\mathscr{S}(\mathscr{C})$ is inner regular (with respect to the system $\left.\left\{\mathscr{S}(\mathscr{C}), \mu^{\prime}, \mathscr{C}, \mathscr{U}\right\}\right)$.

Proof. Let us say that a pair $\left(\mathscr{R}^{\prime}, \mu^{\prime}\right)$ is an admissible extension of $(\mathscr{R}, \mu)$ if $(1) \mathscr{R}^{\prime}$ is a ring such that $\mathscr{R} \subset \mathscr{R}^{\prime} \subset \mathscr{S}(\mathscr{C}),(2) \mu^{\prime}$ is a measure on $\mathscr{R}^{\prime}$ which extends $\mu$ and (3) each set in $\mathscr{R}^{\prime}$ is inner regular (with respect to the system $\left\{\mathscr{R}^{\prime}, \mu^{\prime}, \mathscr{C}, \mathscr{C}\right\}$ ). Notice that $\mu^{\prime}$ is countably additive in view of Theorem 2.3. We order the admissible extensions in the obvious way and observe by Zorn's lemma that there is a maximal admissible extension $\left(\mathscr{R}_{0}, \mu_{0}\right)$. By Lemma 2.4 and the maximality of $\left(\mathscr{R}_{0}, \mu_{0}\right)$, the ring $\mathscr{R}_{0}$ contains $\mathscr{C}$. Moreover, the ring $\mathscr{R}_{0}$ is a sigma-ring by Theorem 2.3. It follows that $\mathscr{R}_{0}=\mathscr{S}(\mathscr{C})$.

By letting the class $\mathscr{C}$ be the class of bounded $n$-closed sets in the locally compact space $X$, we get the following theorem as a consequence of Theorem 2.5:

THEOREM 2.6. Let $\mathscr{S}$ be a sigma-ring containing the Borel sets of $X$ and contained in the $n$-Borel sets of $X$. If $\mu$ is a measure on $\mathscr{S}$ such that each compact set has finite measure and

$$
\mu(E)=\sup \{\mu(C): C \in \mathscr{S} \text { and } C \text { is a bounded n-closed set in } E\}
$$

for each $E \in \mathscr{S}$, then $\mu$ can be extended to an n-regular $n$-Borel measure on $X$. In particular, each regular Borel measure has an n-regular $n$-Borel extension.

Although the extension of a Baire measure to a regular Borel measure is unique [4, Theorem 54.D], there may be more than one extension of an $n$-regular Borel measure to an $n$-regular $n$-Borel measure. For example, let $X$ be the set of all functions from an uncountable set $I$ into the discrete space of two elements $\{0,1\}$, and let $X$ have the product topology (topology of pointwise convergence). Let $\mu$ be normalized Haar measure on the Borel sets of $X$. As in Example 1.2, let us say that two members of $X$ are equivalent if they agree for all but countably many coordinates. If $[x]$ denotes the equivalence class containing $x$, recall that $[x]$ is nearly closed. It can also be seen that $\mu_{i}([x])=0$ and $\mu_{e}([x])=1$ for each $x \in X$. Now 
let $x$ and $y$ be two nonequivalent members of $X$, and let $\mathscr{S}$ be the smallest sigma-ring containing $[x],[y]$ and the Borel sets of $X$. The regular Borel measure $\mu$ can be extended to $n$-regular measures $\mu_{1}$ and $\mu_{2}$ on $\mathscr{S}$ by letting $\mu_{1}([x])=1$ and $\mu_{2}([y])=1$. The measures $\mu_{1}$ and $\mu_{2}$ can in turn be extended to $n$-regular $n$-Borel measures $\mu_{1}^{\prime}$ and $\mu_{2}^{\prime}$. Clearly, $\mu_{1}^{\prime}$ and $\mu_{2}^{\prime}$ are not equal since $\mu_{1}^{\prime}([x])=1$ and $\mu_{2}^{\prime}([x])=$ 0 . Incidentally, it is conceivable that $\mu$ has a unique extension to a translation invariant, $n$-regular $n$-Borel measure on $X$, but I have not been able to show this.

A Borel measure is known to be regular if each bounded open set is inner regular (with respect to the class of compact sets). Similarly, we shall see in Corollary 2.8 that a Borel measure is nregular if each bounded open set is inner regular with respect to the class of bounded $n$-closed sets. For convenience, let us consider classes $\mathscr{C}^{*}$ and $\mathscr{U}^{*}$ which are closed under finite unions and finite intersections, which are subclasses of $\mathscr{C}$ and $\mathscr{U}$, resp., and which satisfy the following axioms (cf. $[1, \S 61]$ ):

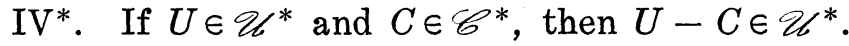

$\mathrm{V}^{*}$. If $C \in \mathscr{C}^{*}$, then there exist $U \in \mathscr{U}^{*}$ and $D \in \mathscr{C}^{*}$ such that $C \subset U \subset D$.

VI*. If $C \in \mathscr{C}^{*}$ and $U \in \mathscr{U}^{*}$, then $C-U \in \mathscr{C}^{*}$.

VII*. The ring $\mathscr{R}$ coincides with $\mathscr{S}\left(\mathscr{C}^{*}\right)$, the sigma-ring generated by $\mathscr{C}^{*}$.

Theorem 2.7. Suppose $\left\{\mathscr{R}, \mu, \mathscr{C}, \mathscr{C}, \mathscr{C}^{*}, \mathscr{C}^{*}\right\}$ is a system satisfying Axioms I-VII and IV*-VII* and that $\mu$ is a countably additive measure on the sigma-ring $\mathscr{R}$. If each "bounded" set in $\mathscr{C}^{*}$ is inner regular, then each set in $\mathscr{R}$ is inner regular (with respect to the system $\{\mathscr{R}, \mu, \mathscr{C}, \mathscr{C}\})$. (Here, "bounded" means "bounded" with respect to $\mathscr{C}^{*}$.)

Proof. Suppose each "bounded" set in $\mathscr{U}^{*}$ is inner regular. We show that if $C$ and $D$ are in $\mathscr{C}^{*}$, then $C-D$ is inner regular (with respect to the system $\{\mathscr{R}, \mu, \mathscr{C}, \mathscr{C}\}$ ). We may assume without loss of generality that $D \subset C$. By Axiom $\mathrm{V}^{*}$ there exists a bounded set $U$ in $\mathscr{C}^{*}$ such that $C \subset U$. By Axiom IV*, $U-C \in \mathscr{U}^{*}$, so that $U-C$ is inner regular by hypothesis. By Theorem 2.1(2), the set $C-D=C \cap(U-D)$ is inner regular also. If $\mathscr{R}\left(\mathscr{C}^{*}\right)$ is the smallest ring containing $\mathscr{C}^{*}$, then each set in $\mathscr{R}\left(\mathscr{C}^{*}\right)$ is inner regular by (1) and (2) of Theorem 2.1. It suffices to show that each "bounded" set in $\mathscr{R}$ is inner regular. Suppose, therefore, that $E$ is a "bounded" set in $\mathscr{R}$. Then there exists a set $K$ in $\mathscr{C}^{*}$ such that $E \subset K$. Let $\mathscr{R}^{K}$ be the set of all $F$ in $\mathscr{R}$ such that $F \cap K$ is inner regular. Clearly, $\mathscr{R}\left(\mathscr{C}^{*}\right) \subset \mathscr{R}^{K}$, and $\mathscr{R}^{K}$ is a monotone class by (3) and (4) 
of Theorem 2.1. Then $\mathscr{R}^{K}=\mathscr{R}$, so that $E \in \mathscr{R}^{K}$, as was to be shown. and

COROLlary 2.8. If $\mu$ is a (countably additive) Borel measure $\mu(U)=\sup \{\mu(C): C \subset U$ and $C$ is a bounded, $n$-closed Borel set $\}$

for all open Borel sets $U$, then $\mu$ is an n-regular Borel measure. If $\mu^{\prime}$ is a (countably additive) n-Borel measure and

$$
\mu^{\prime}(U)=\sup \left\{\mu^{\prime}(C): C \subset U \text { and } C \text { is a bounded n-closed set }\right\}
$$

for all n-open sets $U$, then $\mu^{\prime}$ is an n-regular n-Borel measure.

3. The product of two nearly Borel measures. Throughout this section, $\mu^{\prime}$ and $\nu^{\prime}$ will be $n$-Borel measures on locally compact spaces $X$ and $Y$, resp. Under suitable conditions, we show the existence of an $n$-Borel measure on $X \times Y$ which extends the usual product $\mu^{\prime} \times \nu^{\prime}$. Notice that $M_{x}=\{y \in Y:(x, y) \in M\}$ is an $n$-Borel set in $Y$ whenever $M$ is an $n$-Borel set in $X \times Y$ and $x \in X$.

THEOREM 3.1. If $\nu^{\prime}$ is n-regular and $C$ is a bounded n-closed set in $X \times Y$, then $\nu^{\prime}\left(C_{x}\right)$ as a function in $x$ is measurable with respect to the $n$-Borel sets of $X$.

Proof. The proof is similar to that of [6, Theorem 4.1], where compact sets and open sets are replaced by bounded $n$-closed sets and $n$-open sets, respectively.

THEOREM 3.2. If $\nu^{\prime}$ is purely atomic and $C$ is a bounded $n$-closed set in $X \times Y$, then $\nu^{\prime}\left(C_{x}\right)$ as a function in $x$ is measurable with respect to the $n$-Borel sets of $X$.

Proof. We may assume without loss of generality that $\nu^{\prime}$ takes on the values 0 and 1 only. It suffices to show that $A=\left\{x: \nu^{\prime}\left(C_{x}\right)=1\right\}$ is a bounded $n$-closed set. Clearly $A$ is bounded since $C$ is bounded.

Now suppose $B$ is a countable set in $A$. Then $\nu^{\prime}\left(C_{x}\right)=1$ for all $x \in B$. Let $D=\bigcap\left\{C_{x}: x \in B\right\}$. Then $\nu^{\prime}(D)=1$, and $D$ is contained in $C_{x}$ for all $x \in \operatorname{closure}(B)$. Hence, $\operatorname{closure}(B)$ is a subset of $A$.

Theorem 3.2 shows an advantage of $n$-Borel measures over Borel measures since there exist locally compact spaces $X$ and $Y$, a purely atomic Borel measure $\nu$ on $Y$, and a compact set $K$ in $X \times Y$ such that the function $\nu\left(K_{x}\right)$ is not measurable with respect to the Borel sets of $X$ [5, Examples 1, 2 and 3]. 
It is useful to know when the function $\nu^{\prime}\left(C_{x}\right)$ is measurable with respect to the $n$-Borel sets of $X$ for each bounded $n$-closed set in $X \times Y$; in that case an $n$-Borel measure $\rho_{1}^{\prime}$ can be defined on $X \times Y$ by the formula $\rho_{1}^{\prime}(M)=\int \nu^{\prime}\left(M_{x}\right) d \mu^{\prime}$ for all $n$-Borel sets $M$ in $X \times Y$. (Cf. [6, Theorem 4.4].) By Theorems 3.1 and 3.2, we have the following:

THEOREM 3.3. If the $n$-Borel measure $\nu^{\prime}$ is n-regular or purely atomic, then an $n$-Borel measure $\rho_{1}^{\prime}$ is defined on $X \times Y$ by the formula

$$
\rho_{1}^{\prime}(M)=\int \nu^{\prime}\left(M_{x}\right) d \mu^{\prime}
$$

for all $n$-Borel sets $M$ in $X \times Y$. Similarly, if the $n$-Borel measure $\mu^{\prime}$ is n-regular or purely atomic, then an n-Borel measure $\rho_{2}^{\prime}$ is defined on $X \times Y$ by the formula

$$
\rho_{2}^{\prime}(M)=\int \mu^{\prime}\left(M^{y}\right) d \nu^{\prime}
$$

for all $n$-Borel sets $M$ in $X \times Y$.

If $\mu^{\prime}$ and $\nu^{\prime}$ are both $n$-regular, then $\rho_{1}^{\prime}$ and $\rho_{2}^{\prime}$ are both defined. However, $\rho_{1}^{\prime}$ and $\rho_{2}^{\prime}$ may be unequal and they may both fail to be $n$-regular even though both $\mu^{\prime}$ and $\nu^{\prime}$ are $n$-regular. For example, let $\omega_{1}^{\prime}$ denote the set of ordinals less than or equal to the first uncountable ordinal $\omega_{1}$ and let $\omega_{1}^{\prime}$ have the order topology.

Since $\omega_{1}=\omega_{1}^{\prime}-\left\{\omega_{1}\right\}$ is first countable, the $n$-closed sets of $\omega_{1}^{\prime}$ are precisely the compact sets together with the closed, unbounded subsets of $\omega_{1}$. Hence, the Borel sets and $n$-Borel sets of $\omega_{1}^{\prime}$ coincide. Let $\mu^{\prime}=\nu^{\prime}$ be Dieudonné's nonregular Borel measure on $X=$ $Y=\omega_{1}^{\prime}$. That is, if $E$ is a Borel set in $X$, then $\mu^{\prime}(E)=1$ if $E$ contains a closed, unbounded subset of $\omega_{1}$ and $\mu^{\prime}(E)=0$ otherwise [4, Exercise 52.10]. It is easy to see that the measure $\mu^{\prime}=\nu^{\prime}$ is $n$-regular since each closed, unbounded subset of $\omega_{1}$ is a bounded $n$-closed set in $\omega_{1}^{\prime}$.

If $U=\left\{(x, y) \in X \times Y: x<y<\omega_{1}\right\}$, then $U$ is an $n$-Borel set in $X \times Y$ since it is open in $X \times Y$. It is easy to see that $\rho_{1}^{\prime}(U)=1$ and that $\rho_{2}^{\prime}(U)=0$, so that $\rho_{1}^{\prime}$ and $\rho_{2}^{\prime}$ are unequal in this case. Now if $C$ is an $n$-closed subset of $U$, it is easy to see that $\rho_{1}^{\prime}(C)=0$. The reason is that there exists $x_{0}$ in $\omega_{1}$ such that $C_{x}$ is empty whenever $x>x_{0}$. If this were not the case, then there would exist a sequence $\left\{\left(x_{n}, y_{n}\right)\right\}$ in $C$ such that $x_{n}<y_{n}<x_{n+1}$ for all $n$, and this sequence would converge to a point on the diagonal. Hence, $\rho_{1}^{\prime}$ fails to be 
$n$-regular. Similarly, if $V=\left\{(x, y): y<x<\omega_{1}\right\}$, then $V$ is an open set for which $\rho_{2}^{\prime}(V)=1$. However, $\rho_{2}^{\prime}(C)=0$ for each $n$-closed subset of $V$, so that $\rho_{2}^{\prime}$ also fails to be $n$-regular.

4. Complements on sequentially closed sets. A set $A$ in $X$ is called sequentially closed if the limit of every convergent sequence in $A$ is itself a member of $A$ [2, p. 108]. A set is sequentially open if its complement is sequentially closed. Every $n$-closed set is sequentially closed, but there exist sequentially closed sets which are not $n$-closed. A set will be called sequentially Borel if it is in the smallest sigma-ring containing the class of bounded, sequentially closed sets. It is interesting that the class of bounded, sequentially closed sets behaves much like the class of bounded $n$-closed sets, and we indicate some analogues of the theorems of $\S \S 2$ and 3 for sequentially closed sets and sequentially Borel sets. Recall that a space is sequentially compact if every sequence contains a convergent subsequence.

THeOREM 4.1. If $X$ is sequentially compact and $\left\{C_{i}\right\}$ is a sequence of sequentially closed sets having the finite intersection property, then $\cap C_{i}$ is nonempty.

Proof. Since the intersection of a family of sequentially closed sets is sequentially closed, we may assume without loss of generality that $\left\{C_{i}\right\}$ is a decreasing sequence of nonempty, sequentially closed sets. For each $i$, choose $x_{i} \in C_{i}$. Since $X$ is sequentially compact, some subsequence of $\left\{x_{i}\right\}$ converges, say to $x$. It follows that $x \in C_{i}$ for each $i$, so that $x \in \cap C_{i}$.

Incidentally, the hypothesis that $X$ be sequentially compact is needed in Theorem 4.1. Indeed, the assertion that the class of sequentially closed sets is a compact class is equivalent to the statement that $X$ is sequentially compact.

Suppose $X$ is a locally compact space, $\mathscr{R}$ is a ring containing the Borel sets of $X$ and contained in the sequentially Borel sets, $\mathscr{C}$ is the class of bounded, sequentially closed sets in $X$, and $\mathscr{C}$ is the class of sigma-bounded, sequentially open sets. Axioms I to VII are satisfied for the system $\{\mathscr{R}, \mu, \mathscr{C}, \mathscr{C}\}$. Hence, each Borel [sequentially Borel] set is inner regular with respect to the class of bounded, sequentially closed sets if and only if each Borel [sequentially Borel] set is outer regular with respect to the class of sigma-bounded, sequentially open sets. Indeed, each Borel [sequentially Borel] set is inner regular with respect to the class of bounded, sequentially closed sets if and only if each bounded open set [bounded, sequentially open set] is inner regular with respect to the class of sequentially 
closed sets.

Now suppose that $X$ is also sequentially compact and that $\mathscr{S}$ is a sigma-ring containing the Borel sets and contained in the sequentially Borel sets. In view of Theorem 2.5, we have the following: If $\mu$ is a measure on $\mathscr{S}$ such that each compact set has finite measure and such that each member of $\mathscr{S}$ is inner regular with respect to the sequentially closed sets, then $\mu$ can be extended to a countably additive measure on the sequentially Borel sets such that each sequentially Borel set is inner regular with respect to the class of bounded, sequentially closed sets.

A sequentially Borel measure is a countably additive, nonnegative measure on the sequentially Borel sets such that each compact set has finite measure. We close with an analogue of Theorem 3.3 for the product of two sequentially Borel measures. In a sense, Theorem 4.2 is stronger than Theorem 3.3 since no conditions are placed on the measure $\nu^{\prime}$.

THEOREM 4.2. Suppose $\mu^{\prime}$ and $\nu^{\prime}$ are sequentially Borel measures on locally compact spaces $X$ and $Y$, resp. If $C$ is a bounded, sequentially closed set in $X \times Y$, then $\nu^{\prime}\left(C_{x}\right)$ as a function in $x$ is measurable with respect to the sequentially Borel sets of $X$. Hence, a sequentially Borel measure $\rho_{1}^{\prime}$ is defined on $X \times Y$ by the formula

$$
\rho_{1}^{\prime}(M)=\int \nu^{\prime}\left(M_{x}\right) d \mu^{\prime}
$$

for each sequentially Borel set $M$ in $X \times Y$.

Proof. It suffices to show that the bounded set $A=\left\{x: \nu^{\prime}\left(C_{x}\right) \geqq a\right\}$ is sequentially closed whenever $C$ is a bounded, sequentially closed set in $X \times Y$ and $a$ is positive. Suppose, then, that $\{x(n)\}$ is a sequence in $A$ which converges to $x$. Necessarily, $\lim \sup C_{x(n)}$ is a subset of $C_{x}$. Then since $\nu^{\prime}\left(\lim \sup C_{x(n)}\right) \geqq a[1$, Theorem 17.2], it follows that $x \in A$ and we are done.

\section{REFERENCES}

1. S. K. Berberian, Measure and Integration, Macmillan, New York, 1965.

2. S. P. Franklin, Spaces in which sequences suffice, Fund. Math., 57 (1965), 107-115.

3. S. L. Gulden, W. M. Fleischman and J. H. Weston, Linearly ordered topological spaces, Proc. Amer. Math. Soc., 24 (1970), 197-203.

4. P. R. Halmos, Measure Theory, Van Nostrand, New York, 1950.

5. R. A. Johnson, Measurability of cross section measure of a product Borel set, J. Austral. Math. Soc. Ser. A., 28 (1979), 346-352.

6. R. A. Johnson, On product measures and Fubini's theorem in locally compact spaces, Trans. Amer. Math. Soc., 123 (1966), 112-129.

7. J. Loś and E. Marczewski, Extensions of measure, Fund. Math., 36 (1949), 267-276. 
8. E. Marczewski, On compact measures, Fund. Math., 40 (1953), 113-124.

Received June 20, 1979 and in revised form August 28, 1979.

Washington State University

PUllman. WA 99164 



\section{PACIFIC JOURNAL OF MATHEMATICS}

\section{EDITORS}

DONALD BABBITT (Managing Editor)

University of Galifornia

Los Angeles, California 90024

HUGo RossI

University of Utah

Salt Lake City, UT 84112

C. C. MOORE AND ANDREW OGG

University of California

Berkeley, CA 94720

\section{J. DUGUNDJI}

Department of Mathematics University of Southern California Los Angeles, California 90007

R. FINN AND J. MILGRAM Stanford University Stanford, California 94305

ASSOCIATE EDITORS
E. F. BECKENBACH
B. H. NeumanN
F. WoLF
K. YosHIDA

\section{SUPPORTING INSTITUTIONS}

UNIVERSITY OF BRITISH COLUMBIA UNIVERSITY OF SOUTHERN CALIFONIA CALIFORNIA INSTITUTE OF TECHNOLOGY UNIVERSITY OF CALIFORNIA MONTANA STATE UNIVERSITY STANFORD UNIVERSITY UNIVERSITY OF HAWAII UNIVERSITY OF TOKYO UNIVERSITY OF NEVADA, RENO UNIVERSITY OF UTAH NEW MEXICO STATE UNIVERSITY WASHINGTON STATE UNIVERSITY OREGON STATE UNIVERSITY UNIVERSITY OF OREGON UNIVERSITY OF WASHINGTON 


\section{Pacific Journal of Mathematics \\ Vol. 87, No. $1 \quad$ January, 1980}

Spiros Argyros, A decomposition of complete Boolean algebras ..........

Gerald A. Beer, The approximation of upper semicontinuous multifunctions

by step multifunctions . . . . ....................

Ehrhard Behrends and Richard Evans, Multiplicity theory for Boolean

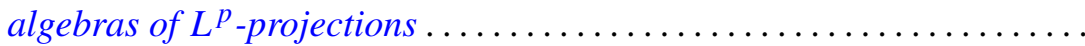

Man-Duen Choi, The full $C^{*}$-algebra of the free group on two

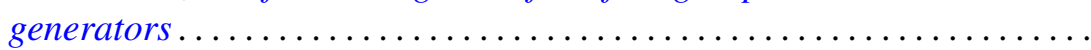

Jen-Chung Chuan, Axioms for closed left ideals in a $C^{*}$-algebra . . . . . . . .

Jo-Ann Deborah Cohen, The strong approximation theorem and locally

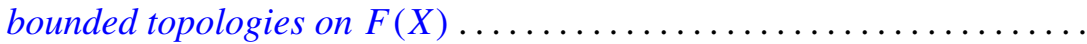

Eugene Harrison Gover and Mark Bernard Ramras, Increasing sequences of Betti numbers............................

Morton Edward Harris, Finite groups having an involution centralizer with

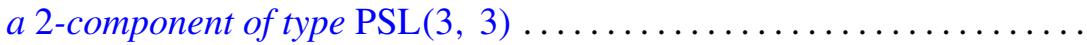

Valéria Botelho de Magalhães Iório, Hopf $C^{*}$-algebras and locally compact

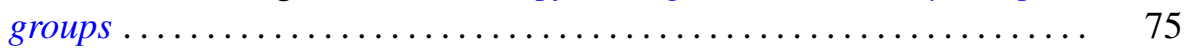

Roy Andrew Johnson, Nearly Borel sets and product measures . . . . . . . . . .

Lowell Edwin Jones, Construction of $Z_{p}$-actions on manifolds . . . . . . . . .

Manuel Lerman and Robert Irving Soare, $d$-simple sets, small sets, and

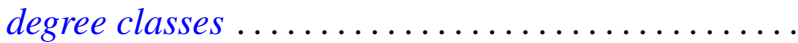

Philip W. McCartney, Neighborly bushes and the Radon-Nikodým property

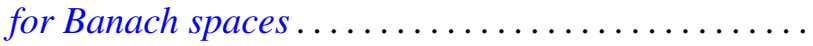

Robert Colman McOwen, Fredholm theory of partial differential equations on complete Riemannian manifolds.

Ernest A. Michael and Carl Preston Pixley, A unified theorem on continuous selections.

Ernest A. Michael, Continuous selections and finite-dimensional sets .

Vassili Nestoridis, Inner functions: noninvariant connected components...

Bun Wong, A maximum principle on Clifford torus and nonexistence of proper holomorphic map from the ball to polydisc.

Steve Wright, Similarity orbits of approximately finite $C^{*}$-algebras . . .

Kenjiro Yanagi, On some fixed point theorems for multivalued

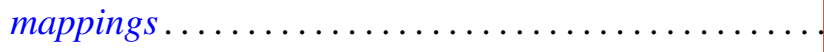

Wieslaw Zelazko, A characterization of LC-nonremovable ideals in commutative Banach algebras 